\title{
Seismic response analysis of Ganga sand deposits using shake table tests
}

\author{
M. Anjali, B. Vivek and P. Raychowdhury*
}

${ }^{*}$ Correspondence: prishati@iitk.ac.in Department of Civil Engineering, Indian Institute of Technology Kanpur, Kanpur, India

\begin{abstract}
Background: An accurate estimation of seismic behavior of a soil deposit is important from various aspects, namely, predicting the influence of the deposit in altering the earthquake waves propagating through that medium, quantifying the amplification or attenuation of the seismic shaking intensities, development of site-specific design spectra for seismic design of infrastructure to be built on that deposit, evaluating the possibility of ground failure and so on.

Method: The present study focuses on dynamic response analysis of local Ganga sand through performing a number of shake table experiments using a flexible laminar container. Uniform, dry Ganga sand of $30 \%$ relative density exhibited acceleration amplification of 20, 40 and $68 \%$ for input motions with 1,2 and $5 \mathrm{~Hz}$ frequencies with amplitudes $0.2,0.36$ and $0.56 \mathrm{~g}$, respectively. Further, analytical investigation on seismic response of the same soil deposit has been carried by assuming the behavior of the soil deposit as: (1) linear and (2) equivalent linear.

Results: The acceleration amplifications are estimated as 1, 4 and $28 \%$ using the linear analysis and 3, 30 and $72 \%$ using the equivalent linear analysis for the 1,2 and $5 \mathrm{~Hz}$ motion, respectively.

Conclusions: Compared to the linear analysis, the equivalent linear analysis predicts the surface amplification of the experiment with better accuracy (a maximum deviation being less than $6.7 \%$ ). This may due to the strain dependent behavior of the soil bed during strong shakings, which is compatible with the assumptions of the equivalent linear analysis procedure.
\end{abstract}

Keywords: Ground response analysis, Shake table tests, Peak ground acceleration, Equivalent linear method, Acceleration amplification, Ganga sand

\section{Introduction}

Ground shaking resulting from earthquake constitutes the major cause of destruction to the built-in environment around the world. During a seismic event, the local site conditions greatly influence the seismic wave characteristics such as the acceleration amplitude and the frequency content. For example, in 1985 Mexico City Earthquake, the Mexico City had experienced enormous devastation in spite of being $350 \mathrm{~km}$ from the epicentre, mainly because of site amplifications of alluvial soft deposits. Similarly, San Francisco bay area had experienced large ground amplification during 1989 Loma Prieta Earthquake largely due to site amplification. Events such as 1994 Northridge earthquake,

(C) 2015 Anjali et al. This article is distributed under the terms of the Creative Commons Attribution 4.0 International License (http:// creativecommons.org/licenses/by/4.0/), which permits unrestricted use, distribution, and reproduction in any medium, provided you give appropriate credit to the original author(s) and the source, provide a link to the Creative Commons license, and indicate if changes were made. 
1995 Kobe earthquake and 1999 Chi-Chi earthquake have also demonstrated the relevance of local geologic and topographic conditions on the ground response.

Over the past few decades, significant research has been carried out to develop quantitative methods to predict the influence of local site conditions on strong ground motions. Some of these studies adopted analytical methods, whereas others involved experimental measures. Observations of some of these investigations are adopted in the current design practice to develop guidelines for site-specific design spectra. A brief discussion on the previous work done in this area is provided herein.

In a pioneering work, Seed and Idriss [1] proposed an approximate linear solution by assuming constant values of soil properties during an earthquake. Schnabel et al. [2] developed a method for one-dimensional ground response analysis using equivalent linear approach, which was implemented in widely accepted ground response software SHAKE. Ishibashi and Zhang [3] reanalyzed the available experimental data on dynamic properties of soil, such as strain-dependant shear modulus and damping ratio, and proposed a few generalized formula for predicting those properties. Elgamal et al. [4] utilized free-field downhole-array seismic records to identify and model the recorded response of sites in Lotung, Taiwan and Treasure Island, California. In a subsequent work by Zeghal et al. [5], the same information is used to model the liquefaction behavior of the above-mentioned sites. Borja et al. [6] developed a fully nonlinear finite-element model to investigate the impact of hysteretic and viscous material behavior on the down-hole motion recorded by an array at a large-scale seismic test site in Lotung, Taiwan during an earthquake event. Finn et al. [7] examined the applicability of 1-D and 2-D site response analyses for amplification studies in Fraser Delta, British Columbia using recorded ground motions from 1996 Duvall earthquake. Kwok et al. [8] performed a comprehensive study on parameter and usage protocols for using various nonlinear and equivalent linear ground response analysis approaches. Jafarzadeh et al. [9] examined the response of Babolsar dry sand subjected to harmonic motions in a shake table test and numerical analysis. Turan et al. [10] performed a series of shaking table tests to study the nonlinear seismic behavior of clay using a laminar box. Phillips and Hashash [11] developed two frequency dependant viscous damping formulations suitable for nonlinear 1-D site response analysis for a wide range of strains. Jishnu et al. [12] performed analytical ground response analysis of Kanpur soil using 1-D and 2-D equivalent linear analysis to predict ground acceleration and liquefaction potential of the region. Hokmabadi et al. [13] have performed shake table tests using laminar box concept to evaluate response of soil-pile-structure interaction of soft soils.

It may be noted that seismic response of Ganga sand deposits, which covers a large area along Indo-Gangetic plane of India, has not been investigated thoroughly. Although a few static element level tests on Ganga sand were performed in the past, however, comprehensive dynamic characterization of the same using shake table experiment coupled with equivalent-linear analysis has not been done before. The present study focuses on dynamic response analysis of local Ganga Sand through performing a number of shake table experiments using a flexible laminar container. Further, the experimental observations are compared with 1-D linear elastic and equivalent-linear ground response analysis results. 


\section{Experimental investigation}

To investigate the seismic response of Ganga sand through shake table experiments, a laminar soil box has been used. The laminar box has its advantage over conventional rigid boxes in reducing the boundary effects. The following subsections describe the basic geotechnical characterization of the soil, experimental set up, instrumentation, input excitations, and the observations made from the experiments.

\section{Basic characterization of Ganga sand}

Ganga sand covers a wide area of Northern and Eastern India. Prior to performing the shake table experiments and analytical modeling, some basic geotechnical tests including sieve analysis, hydrometer tests, specific gravity tests, and direct shear tests have been carried out in order to characterize the soil. As per unified soil classification system according to ASTM D2487-11 [14], the soil is classified as poorly graded sand with low fine content. The minimum and maximum void ratios of the sample are found as 0.712 and 0.936 , respectively. The present study considers a loose soil deposit, with a $30 \%$ relative density. Direct shear test has been carried out on the sample to estimate the shear strength parameters. Three sets of tests have been done under normal stresses of 50, 100 and $150 \mathrm{kPa}$, respectively. Linear regression between applied normal stress and obtained peak shear stress data points indicated a friction angle of $33^{\circ}$ (Fig. 1). The geotechnical properties of the soil obtained from the above-mentioned tests are tabulated in Table 1.

\section{Shake table test setup}

A series of shake table tests has been carried out on the dry Ganga sand of $30 \%$ relative density after the sand is placed in a flexible laminar box. The flexible laminar box consists of five horizontal square shaped lamina of size $300 \mathrm{~mm} \times 300 \mathrm{~mm} \times 27 \mathrm{~mm}$ as shown in Fig. 2a. Each lamina is supported individually by six low-friction roller bearings (three per side), which are guided through a guide channel. Multiple roller bearings considerably reduce the friction between the lamina. The guide channels are connected to the external frame which transfers the weight of the lamina away from the shake table. A clearance of $3 \mathrm{~mm}$ is provided between the individual lamina to establish movement of individual lamina. The roller bearing system allows the movement of the container to the direction of the motion of the shake table. In order to place the soil inside the flexible
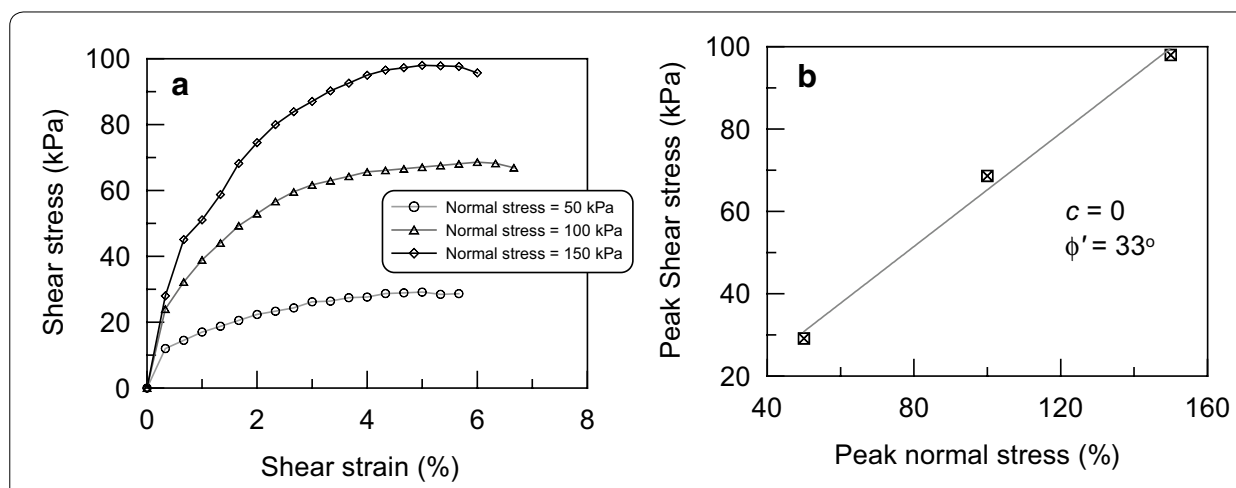

Fig. 1 Results of direct shear test on the Ganga sand: a shear stress vs. shear strain and $\mathbf{b}$ peak shear stress vs. normal stress 
Table 1 Geotechnical properties of Ganga sand

\begin{tabular}{ll}
\hline Property & Value \\
\hline Soil Type & Poorly graded sand (SP) \\
Specific gravity, $G_{S}$ & 2.70 \\
Coefficient of uniformity, $C_{u}$ & 1.5 \\
Coefficient of curvature, $C_{c}$ & 1.1 \\
Sand content (\%) & 85.40 \\
Silt content (\%) & 12.92 \\
Clay content (\%) & 1.68 \\
Maximum void ratio, $e_{\max }$ & 0.936 \\
Minimum void ratio, $e_{\min }$ & 0.712 \\
Cohesion (kPa) & 0 \\
Friction angle, $\phi^{\prime}$ & $33^{\circ}$ \\
\hline
\end{tabular}

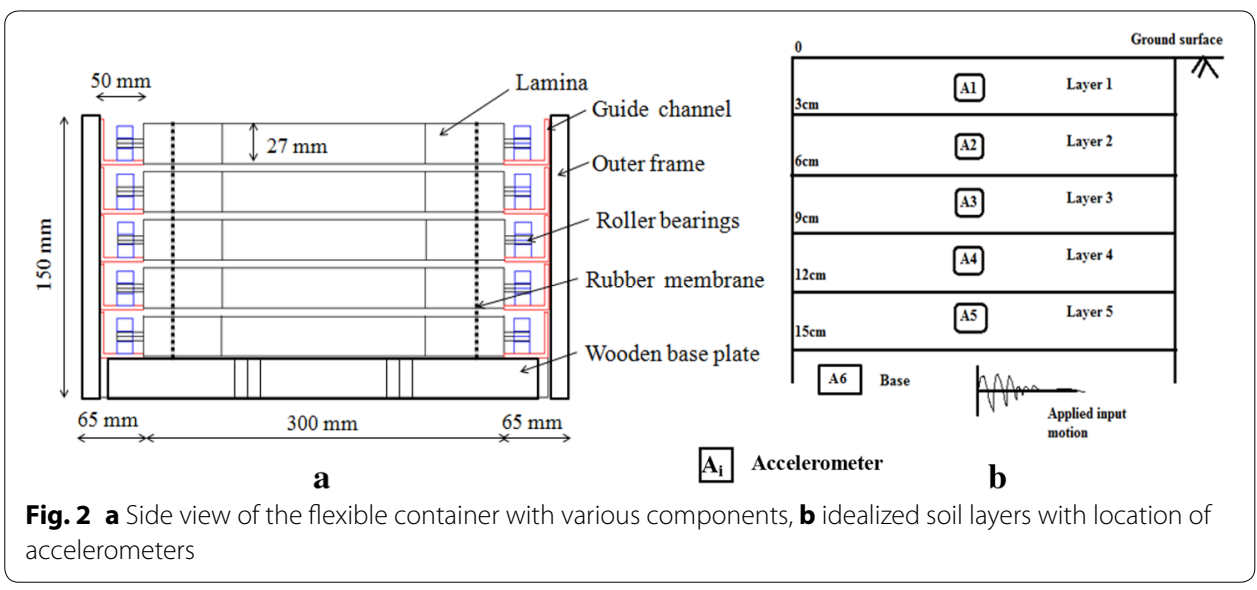

laminar box, a wooden base plate of size $300 \mathrm{~mm} \times 300 \mathrm{~mm} \times 5 \mathrm{~mm}$ is bolted to the top of the shake table. The top of the wooden base plate is epoxied with course sand to minimize the sliding movement at the soil and base plate interface.

Five accelerometers (A1 to A5) have been attached to each individual lamina of the flexible box to measure the acceleration response at different depths. Another accelerometer A6 was attached to the wooden base plate to measure the input motion. The locations of the accelerometers are as shown in Fig. $2 \mathrm{~b}$.

The interior of the flexible laminar box is lined with a thin rubber membrane to prevent the soil spill out through the gaps between the lamina. The thickness of the rubber membrane is $0.3 \mathrm{~mm}$ and its compression modulus is $0.04 \mathrm{~N} / \mathrm{mm}$. This provides a minimum interference with the flexibility of the box. A photograph of the test setup is shown in Fig. 3.

The small shaking table at Indian Institute of Technology, Kanpur comprises $150 \mathrm{~mm} \times 150 \mathrm{~mm}$ table with a capacity of 5.26 ton which can move uniaxially through electrical actuators controlled by digital control module. The flexible shear box is fixed to the shake table and the dry Ganga sand sample is placed in the box at a relative density of $30 \%$. The sand is layered inside the container through the well-established rainfall pouring technique by hand hopper to maintain the desired relative density. To obtain 


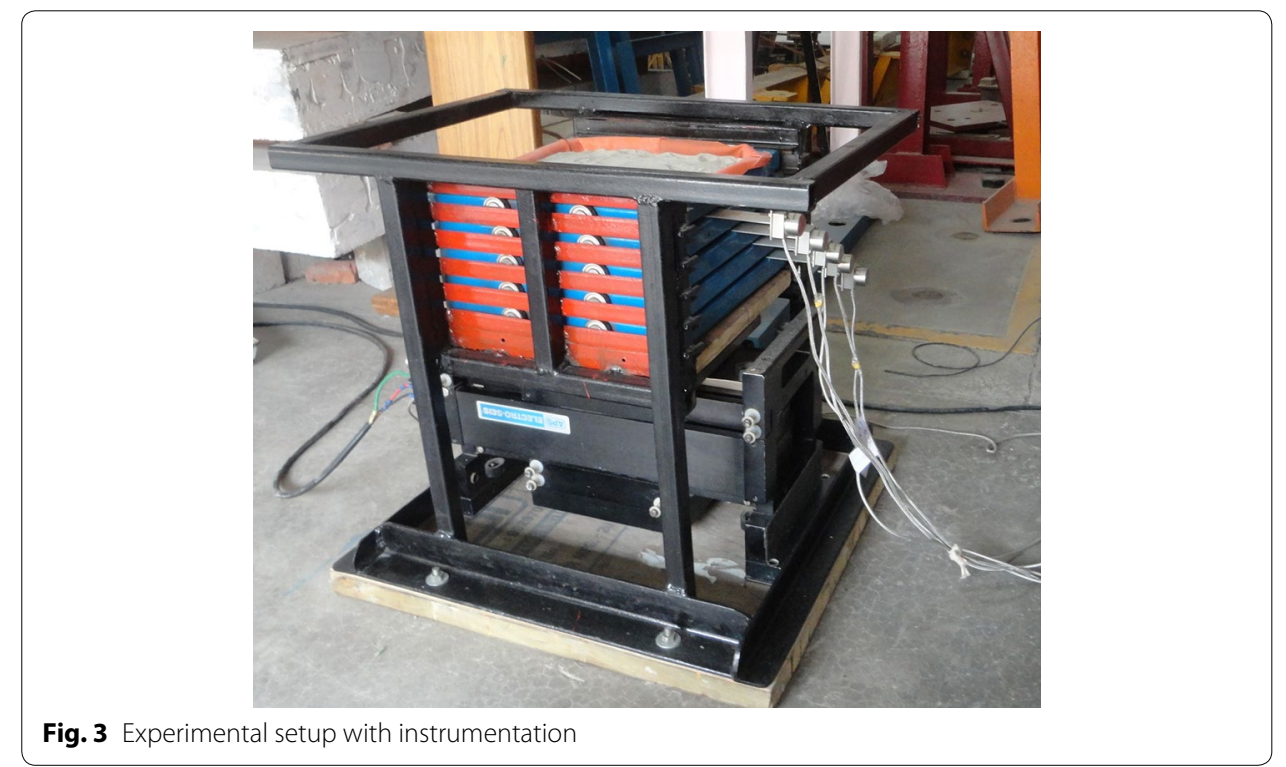

the uniformity of the soil bed, the hand hopper is swung back and forth like a pendulum, keeping a constant height of fall that provides a uniform deposition over the layers. The height of the fall to achieve the relative density for the dense and loose sand is calculated after Shubham et al. [15], which was established through a series of pluviation tests. In order to assure the uniformity of sand deposition while filling, a dynamic cone penetrometer is employed at different location along the horizontal direction.

\section{Input excitations}

Three sinusoidal input excitations with different amplitudes and frequencies have been used in this study. The details of these motions are given in Table 2. Accelerometer A6 is attached to the shake table for measuring the base acceleration that are equivalent to the bedrock motion of a deposit. The time histories of the input excitations are shown in Fig. 4. Note that although the motions used in the experiments had durations of about $80 \mathrm{~s}$, the time histories in Fig. 4 only shows the acceleration cycles up to the first $5 \mathrm{~s}$ for clarity.

\section{Experimental results}

The accelerometers A1 to A5 located at varying depths, as shown in the Fig. $2 \mathrm{~b}$ are used to measure the acceleration time histories of the soil during the excitations. In order to process the data, the acceleration recordings are sequentially filtered using a 10th order low pass Butterworth filter (with a cutoff frequency of $10 \mathrm{~Hz}$ ) and 4th order high pass

Table 2 Details of input excitations

\begin{tabular}{lll}
\hline Input motion & Amplitude (in g) & Frequency (Hz) \\
\hline Motion 1 & 0.20 & 1 \\
Motion 2 & 0.36 & 2 \\
Motion 3 & 0.54 & 5 \\
\hline
\end{tabular}



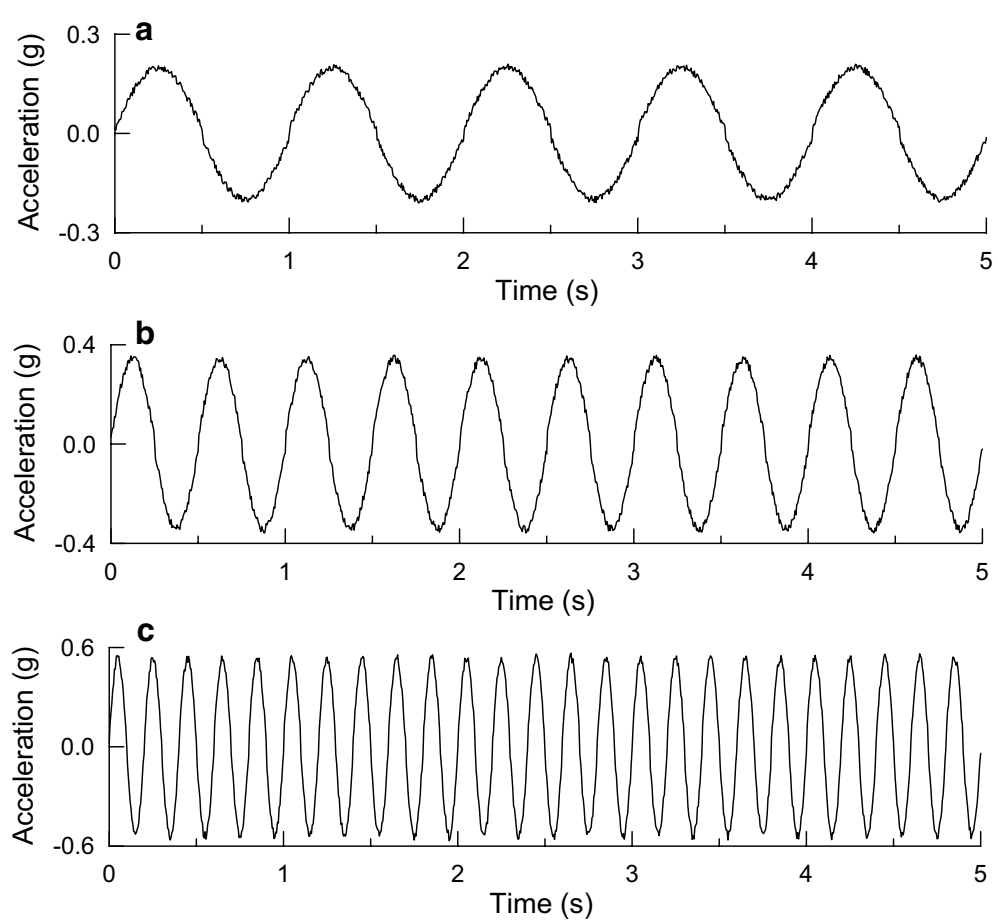

Fig. 4 Input excitations applied at the base of the soil box: a motion 1, b motion 2 and $\mathbf{c}$ motion 3 for the shake table experiment on Ganga sand

filter to eliminate minor contributions of high and low frequency values in the acceleration recordings.

Figure 5 shows the acceleration responses at various locations of the soil bed. Records of accelerometer A1, A3 and A5, indicating responses at layer 1, 3 and 5 are shown here. It can be clearly observed that as the waves travel from the bottom to top of the deposit, acceleration amplification is taken place for all cases. This is an indication of shear wave propagation through the soil medium and consequent increase in strain. It is observed from Fig. 5a that although the acceleration is not significantly amplified for the lowest intensity motion (motion 1), a complete phase difference is evident between the top and other layers. For moderate intensity motion (motion 2), higher amplification is observed (Fig. 5b), whereas the highest intensity motion (motion 3 ) exhibits a significant amplification as shown in Fig. 5c. A comparison of the peak accelerations of top layer of the bed and base of the box indicated 20, 40 and $62 \%$ amplification for motion 1, 2 and 3 , respectively. These amplifications resulted from the propagation of the shear waves from the base to the top surface of the soil bed. It can be concluded that a homogeneous deposit of dry Ganga sand is expected to experience as much as $62 \%$ acceleration amplification when subjected to an earthquake motion of intensity $0.54 \mathrm{~g}$.

\section{Analytical investigation}

Since the soil box is small, in order to effectively use the experimental results for predicting the response of a prototype deposit, well-justified similitude rules have to be adopted. The present study utilizes the similitude rule established by Iai [16], where 

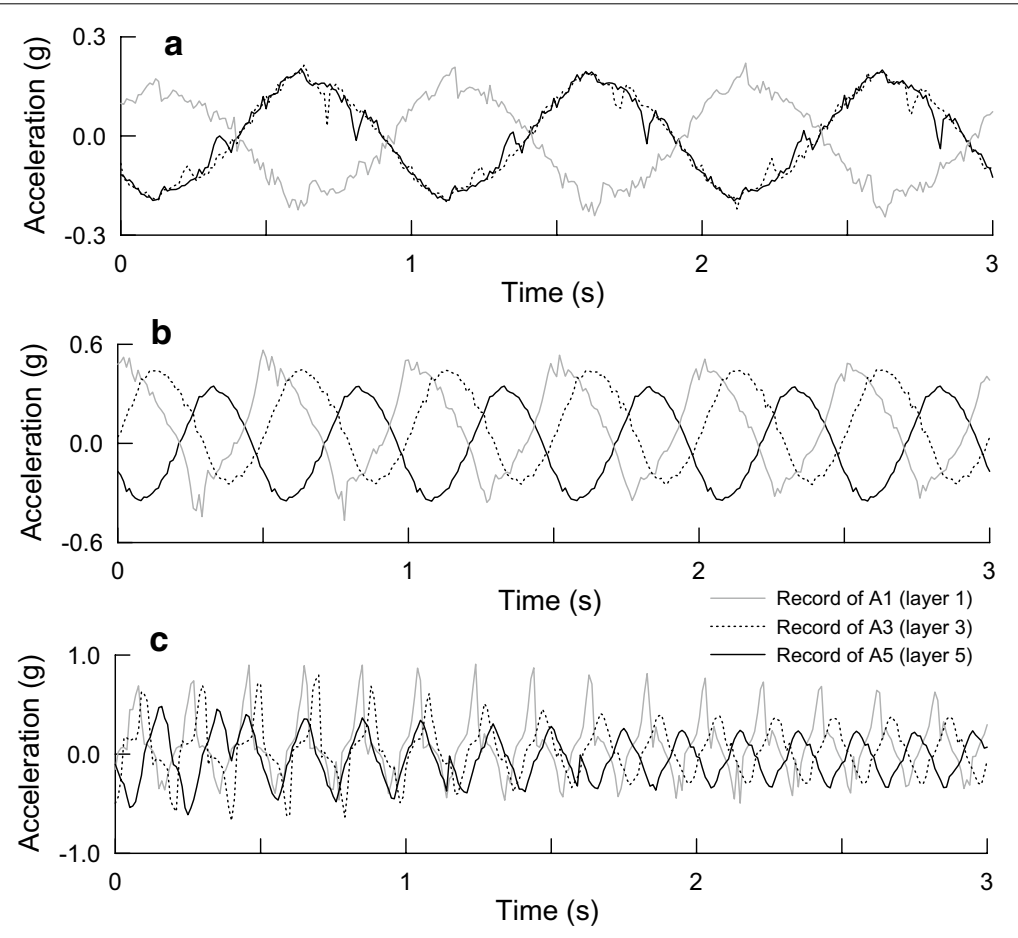

Fig. 5 Acceleration records of accelerometers at various depths during shake table experiment on Ganga sand for: a motion 1, b motion 2 and $\mathbf{c}$ motion 3

the scaling problem is defined in terms of geometry, density and strain scaling factors. The model developed by Iai [16] assumes a continuous soil medium and maintains the equilibrium at low strain deformations, which is in good agreement to the nature of the present study. Table 3 provides the scaling relationship between the prototype and the model. The geometric scaling factor, $\beta$ is considered to be 20 in the present study. The seismic response of the Ganga sand deposit has been carried out using one-dimensional ground response analysis adopting (1) linear elastic approach and (2) equivalent linear approach. Followed are brief descriptions of each method.

Table 3 Similitude relationship between the prototype and the model (after lai [13])

\begin{tabular}{ll}
\hline Physical parameter & Scaling factor \\
\hline Length & $\beta$ \\
Mass density & 1 \\
Strain & 1 \\
Stress & $\beta$ \\
Time & $\beta^{0.5}$ \\
Frequency & $\beta^{-0.5}$ \\
Acceleration & 1 \\
Shear wave velocity & $\beta^{0.5}$ \\
Shear modulus & $\beta$
\end{tabular}




\section{Linear elastic ground response analysis}

In the 1-D ground response analysis, it is assumed that the domain is comprised of horizontal boundaries (horizontal ground surface and bedrock) of infinite extend and the input motion is imposed as horizontally polarized vertically propagating $\mathrm{SH}$ wave originated from the bedrock. It is also assumed that the soil possesses linear elastic properties and remains constant along the thickness of each layer. Each layer is assumed to behave as Kelvin-Voigt element, where the total resistance of the soil layer to shearing deformation is provided by sum of an elastic spring component and a viscous damper component. If the bedrock is subjected to a horizontal harmonic sine wave motion with circular frequency $\omega$, the wave equation of a soil medium can be expressed as (Kramer [17]):

$$
\rho \frac{\partial^{2} u}{\partial t^{2}}=G \frac{\partial^{2} u}{\partial z^{2}}+\eta \frac{\partial^{3} z}{\partial^{2} z \cdot \partial t}
$$

where, $u=u(z, t)$ denotes horizontal displacement of soil medium at any depth $z$ at time $t, \rho$ is mass density, $G$ is shear modulus, and $\eta$ is equivalent viscosity of soil medium which can be expressed in terms of $G, \omega$ and hysteretic damping ratio, $\xi$

$$
\eta=\frac{2 G}{\omega} \xi
$$

The equation of motion has the solution given by

$$
u(z, t)=A e^{i\left(\omega t-k^{*} z\right)}+B e^{i\left(\omega t+k^{*} z\right)}
$$

where, A and B represents the amplitudes of upward and downward travelling waves and $k^{*}$ is the complex wave number expressed as a function of wave number $k$ and damping ratio $\xi$.

$$
k^{*}=k(1-i \xi)
$$

By imposing boundary condition that the shear stress at the ground surface is zero, we get

$$
\tau(z=0, t)=G \frac{d u(z=0, t)}{d z}=0
$$

Comparing Eqs. (3) and (5), we get, $A=B$. Hence, the horizontal displacement can be represented as:

$$
u(z, t)=2 A \cos k^{*} z e^{i \omega t} .
$$

The transfer function or amplification function that relates the amplitude of the motion at the bedrock and the ground surface can be expressed as

$$
F(\omega)=\frac{u_{\max }(z=0, t)}{u_{\max }(z=H, t)}=\frac{1}{\cos ^{*} H}
$$

where, $\mathrm{H}$ is the total thickness of the soil deposit. For small value of $\xi$, modulus of $F(\omega)$ can be approximated as 


$$
|F(\omega)|=\frac{1}{\sqrt{\cos ^{2}(k H)+(\xi \mathrm{kH})^{2}}}
$$

Replacing the wave number $k$ with $\omega / v_{s}$ ( $\mathrm{v}_{\mathrm{s}}$ being the shear wave velocity) the following expression for the transfer function may be established for a homogeneous damped soil deposit

$$
|F(\omega)|=\frac{1}{\sqrt{\cos ^{2}\left(\omega H / v_{s}\right)+\left(\xi \omega H / \nu_{s}\right)^{2}}}
$$

As evident from Eq. (9), the transfer function is dependent on input frequency, shear wave velocity (or shear modulus), damping ratio and thickness of the deposit. Figure 6a, $\mathrm{b}$ demonstrate the variation of transfer function with shear modulus and damping ratio, respectively. It is evident from Eq. (9) as well as from Fig. 6 that while damping ratio has significant effect on the magnitude of the transfer function, shear modulus has considerable influence on the natural frequency and modal properties of a deposit. It can be seen from Fig. 6a that a reduction from 15 to $8 \mathrm{MPa}$ in shear modulus has shifted the natural frequency of the deposit from 52 to $38 \mathrm{rad} / \mathrm{s}$, whereas Fig. $6 \mathrm{~b}$ indicates that an increase in damping ratio from 5 to $10 \%$ reduces the amplification from 12 to 6 at the fundamental frequency.

To simulate the behavior of the deposit considered in the present study, the low-strain elastic shear modulus of the soil has been calculated based on empirical relationship after Seed and Idriss [18]:

$$
G=1000 K_{2, \max }\left(\sigma_{m}^{\prime}\right)^{0.5}
$$

where, $K_{2, \max }$ is a function of void ratio and relative density, and $\sigma_{m}^{\prime}$ is the mean effective stress in $\mathrm{lb} / \mathrm{ft}^{2}$. For a loose sand of relative density $30 \%, K_{2, \max }$ is estimated as 34 (after Seed and Idriss [18]). Since the soil during the experiment was not subjected to any confinement, therefore, the shear modulus for numerical analysis has been estimated for a very low mean effective stress of $1.0 \mathrm{kPa}$. The low-strain shear modulus is estimated as 7.5 MPa using the above-mentioned method and assumptions. A damping of $5 \%$ is assumed for the numerical analysis. For the above-mentioned value of shear modulus, the shear wave velocity and the natural frequency of the deposit are estimated as $70.71 \mathrm{~m} / \mathrm{s}$ and $11 \mathrm{~Hz}$.
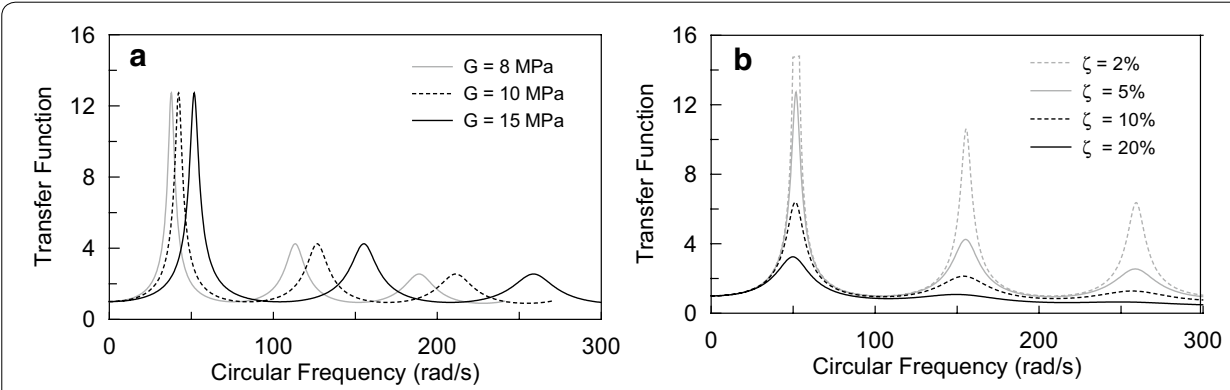

Fig. 6 Variation of transfer functions with $\mathbf{a}$ shear modulus and $\mathbf{b}$ damping ratio 


\section{Equivalent linear ground response analysis}

It is well-known that soil deposits when subjected to strong motions exhibit nonlinear behavior. In order to account for nonlinearity of soil during shaking, an equivalent linear method is adopted herein. The equivalent-linear method is widely being used in the design industry and the method is capable of considering effect of inelasticity through modulus reduction and damping increment.

In this method, strain-dependent secant shear modulus and damping ratios are used, and the response of each layer is obtained through an iterative procedure until the assumed properties are compatible with computed strain levels in all layers. Widely used computer program SHAKE 2000 [2] has been utilized for this analysis. The average sand curves after Seed and Idriss [18] are used to model the modulus reduction and damping ratio relationship with shear strain (Fig. 7).

In both linear and equivalent linear analysis, the soil deposit is divided into 5 equal layers to be consistent with the experimental set-up. Sinusoidal motions similar to the experimental input are applied at the bedrock, and responses at each layer are determined and compared with each other. The following section provides the comparison between the experimental and analytical results.

\section{Comparison between experiment and analysis}

Figure $8 \mathrm{a}-\mathrm{c}$ present the acceleration time histories at the ground surface obtained from experiment and analysis for motion 1, 2 and 3, respectively. It may be observed that the phase of the experimental response is slightly different than the analytical ones. The amplitudes of experiment and analyses are in good agreement for motion 1 and 2, however, for the third motion, the experimental results is skewed and deviate largely from the analytical response. This deviation may be resulted from the nonlinear behavior of the soil bed during the high intensity excitation.

Figure 9 shows the peak accelerations along the depth of the soil bed. The depth of each record is represented as a ratio of the total thickness of deposit (i.e. normalized depth). It may be observed that in general, all methods and all motions show acceleration amplification for this soil type. Moreover, for all cases, the amplification increases with increasing distance from the bedrock. For motion 1, the experiment and both linear and nonlinear analysis are in good agreement until middle of the deposit and then starts
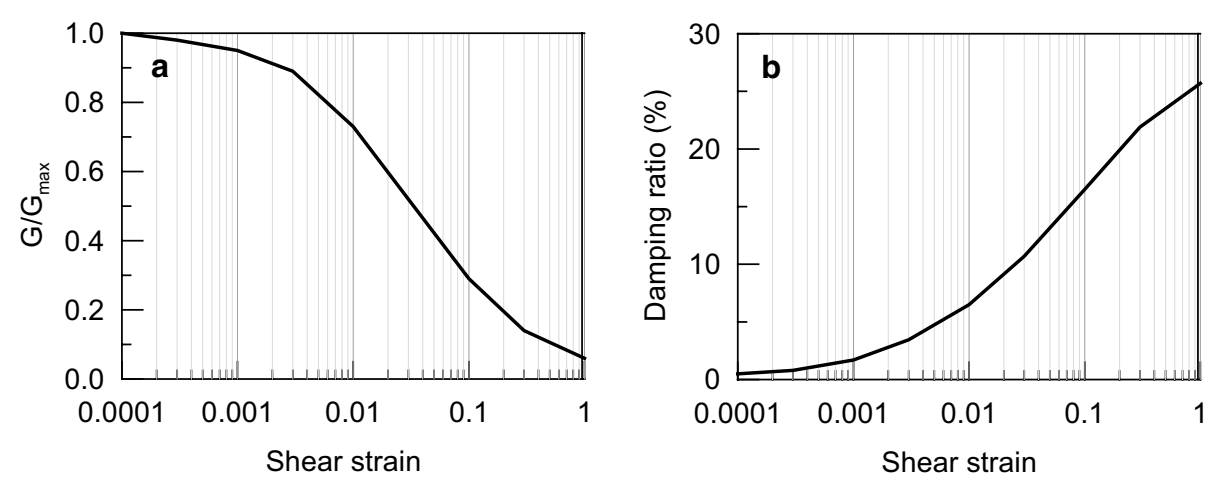

Fig. 7 Dynamic soil properties of sand used in the SHAKE-2000 analysis: a modulus reduction curve and $\mathbf{b}$ damping ratio curve (after Seed and Idriss [15]) 

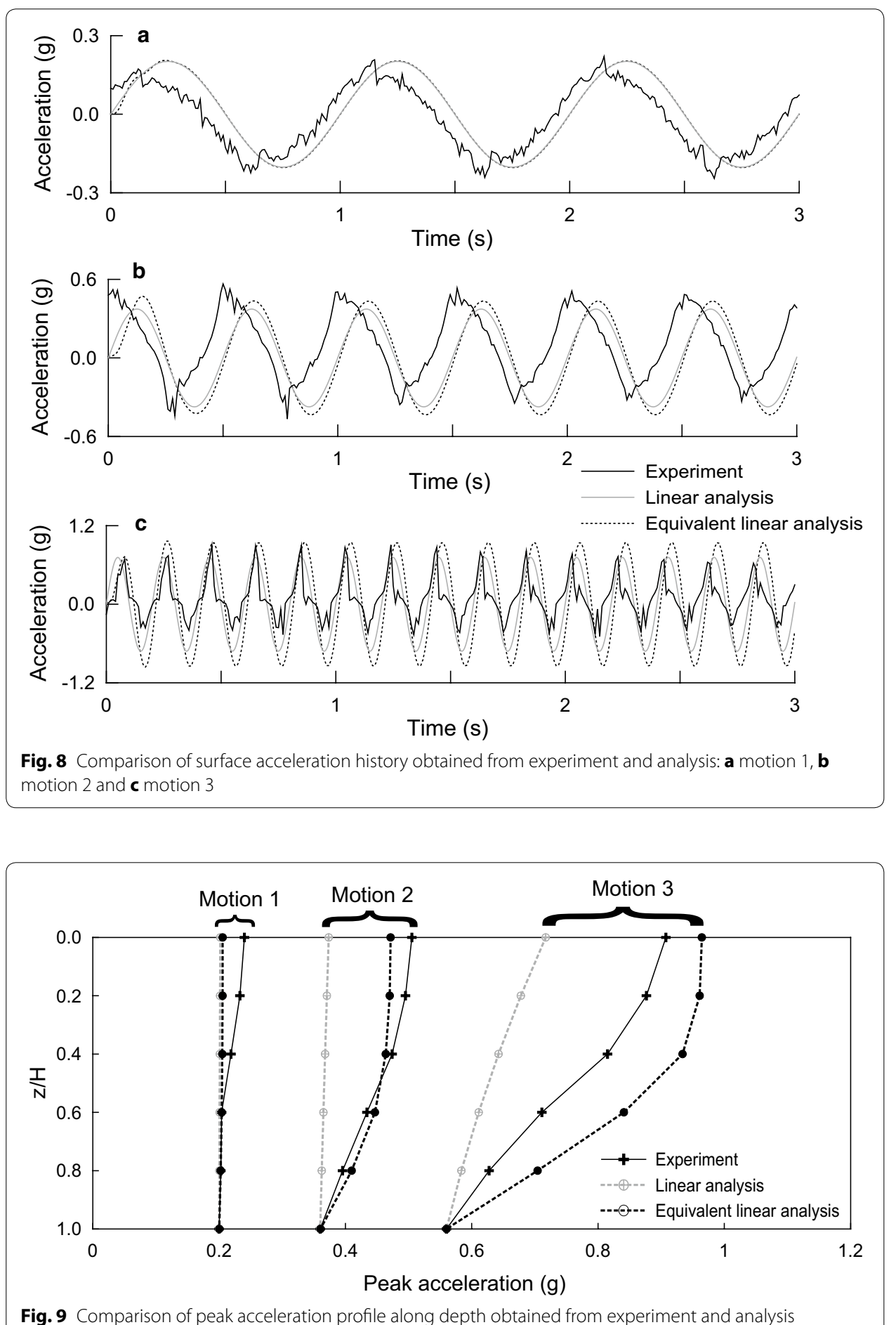

deviating towards the top of the deposit. The peak ground acceleration (PGA) obtained from linear analysis, equivalent linear analysis and experiment are 0.202, 0.206 and $0.24 \mathrm{~g}$, respectively for this input motion. For motion 2, linear analysis largely underestimates the response, but the equivalent linear method is able to capture the accelerations 
obtained from the experiment reasonably well. The PGA obtained from linear analysis, equivalent linear analysis and the experiment are $0.37,0.47$ and $0.51 \mathrm{~g}$, respectively for motion 2. This indicates an underestimation of $26 \%$ by the linear method and $6.7 \%$ by the equivalent linear method to capture the experimental PGA. For motion 3, the linear method still underestimates the acceleration response of the experiment, but the equivalent linear method has found to slightly overestimate the same. The extent of underestimation of PGA is $21 \%$ by the linear method, whereas the overestimation of the same is $6.26 \%$ by the equivalent linear method. The estimated PGAs are $0.71,0.96$, and $0.908 \mathrm{~g}$ using linear, equivalent linear and experiment, respectively for this motion.

The amount of acceleration amplifications at various depths for different input motions using linear analysis, equivalent linear analysis and the experiment are summarized in Table 4. It may be noted that with increasing intensity and frequency of the motions, the amplification of acceleration has an increasing trend for both the analysis methods as well as the experimental observations. It may be noted that the amplification function when estimated using a linear method, depends only on frequency of the input motion and not on the amplitude of the motion. However, in the equivalent linear method, strain-dependant shear modulus and damping are used; hence, the intensity of the motion plays an important role in estimating the amplifications. The latter case is a better representation of the real scenario of soil deposits subjected to earthquakes, as it is well-known that soil indeed exhibits strain-dependant behavior upon shearing at high strain levels. This has been reflected in the comparison results, and it has been observed that compared to the linear analysis, the equivalent linear method predicts the acceleration amplification of the experiment with better accuracy.

\section{Conclusions}

It is well-known that local site conditions greatly influence the acceleration amplitude and frequency characteristics of seismic waves during an earthquake. An appropriate ground response analysis is therefore crucial for assessment of seismic demand of structures built on it, development of realistic site-specific design spectra, estimation of dynamic shear stress-strain behavior, evaluation of liquefaction potential, and so on. The present study focuses on seismic response analysis of locally available dry Ganga sand with $30 \%$ relative density using both experimental and analytical methodologies. The experimental investigation includes shake table experiments using a flexible laminar container, whereas the analytical component involves 1-D dynamic ground response analysis using linear approach and equivalent linear approach. The experimental results clearly indicate that the base excitations get amplified significantly when travelled through the soil bed during the shaking. The amplifications are observed as 20, 40 and $62 \%$ for motion 1,2 , and 3, respectively. The acceleration amplifications are estimated

Table 4 Acceleration amplification from bedrock to surface

\begin{tabular}{llll}
\hline Motion & Experiment & Linear analysis & Equivalent linear analysis \\
\hline 1 & 1.20 & 1.01 & 1.03 \\
2 & 1.40 & 1.04 & 1.31 \\
3 & 1.62 & 1.28 & 1.72 \\
\hline
\end{tabular}


as 1, 4 and $28 \%$ using the linear analysis and for are 3, 30 and $72 \%$ using the equivalent ground response analysis for motion 1, 2 and 3, respectively. Compared to the linear analysis, the equivalent linear analysis predicts the surface amplification of the experiment with better accuracy (a maximum deviation being less than $6.7 \%$ ). This may due to the strain dependent behavior of the soil bed during strong shakings, which is compatible with the assumptions of the equivalent linear analysis procedure.

Authors' contributions

MA and BV conducted the shake table experiments and carried out the analysis. PR had given the research idea, helped in performing analysis and participated in drafting the manuscript. All authors read and approved the final manuscript.

\section{Acknowledgements}

The work was funded by Department of Science and Technology, India under the project number SB/FTP/ETA-320/2012. Any opinions, findings, and conclusions or recommendations expressed in this paper are those of the authors, and do not necessarily reflect those of the funding organization.

\section{Compliance with ethical guidelines}

\section{Competing interests}

The authors declare that they have no competing interests.

Received: 16 January 2015 Accepted: 7 September 2015

Published online: 23 September 2015

\section{References}

1. Seed HB, Idriss IM (1969) Influence of soil conditions on ground motions during earthquakes. J Soil Mech Found Div ASCE 95(1):99-137

2. Schnabel BP, Lysmer J, Seed HB (1972) SHAKE: a computer program for earthquake response analysis of horizontallylayered sites. Earthquake Engineering Research Center, University of California at Berkeley, Berkeley

3. Ishibashi I, Zhang X (1993) Unified dynamic shear moduli and damping ratios of sand and clay. Soils Found 33(1):182-191

4. Elgamal AW, Zeghal M, Parra E, Gunturi R, Tang HT, Stepp JC (1996) Identification and modeling of earthquake ground response-I. Site amplification. Soil Dyn Earthq Eng 15:523-547

5. Zeghal M, Elgamal AW, Parra E (1996) Identification and modeling of earthquake ground response-II. Site liquefaction. Soil Dyn Earthq Eng 15:523-547

6. Borja RI, Chao HY, Montáns FJ, Lin CH (1999) Nonlinear ground response at Lotung LSST Site, ASCE. J Geotech Geoenviron Eng 125(3):187-197

7. Finn WDL, Zhai E, Thavaraj T, Hao XS, Ventura CE (2003) 1-D and 2-D analyses of weak motion data in Fraser Delta from 1966 Duvall earthquake. Soil Dyn Earthq Eng 23(4):323-329

8. Kwok AOL, Stewart JP, Hashash YMA, Matasovic N, Pyke R, Wang Z, Yang Z (2007) Use of exact solutions of wave propagation problems to guide implementation of nonlinear seismic ground. ASCE J Geotechn Geoenviron Eng 133(11):1385-1398

9. Jafarzadeh F, Faghihi D, Ehsani M (2008) Numerical Simulation of Shaking Table Tests on Dynamic Response of Dry Sand. In: 14th World Conference on Earthquake Engineering, October 12-17, 2008, Beijing, China

10. Turan A, Hinchberger SD, Naggar HE (2009) Design and commissioning of a laminar soil container for use on small shaking tables. Soil Dyn Earthq Eng 29:404-414

11. Phillips C, Hashash YMA (2009) Damping formulation for nonlinear 1-D site response analyses. Soil Dyn Earthq Eng 29:1143-1158

12. Jishnu RB, Naik SP, Patra NR, Malik JN (2013) Ground response analysis of Kanpur soil along indo-gangetic plains. Soil Dyn Earthq Eng 51:47-57

13. Hokmabadi AS, Fatahi B, Samali B (2015) Physical modeling of seismic soil-pile-structure interaction for buildings on soft soils. Int J Geomech 15(2):04014046

14. ASTM D2487-11 (2015) Standard Practice for Classification of Soils for Engineering Purposes (Unified Soil Classification System). American Society of Testing and Materials, West Conshohocken, PA, USA

15. Shubham S, Srinivasan V, Ghosh P (2014) Effective utilization of dynamic penetrometer in determining the soil resistance of the reconstituted sand bed. In: The 15th Asian Regional Conference on Soil Mechanics and Geotechnical Engineering, Fukuoka, Japan

16. lai S (1989) Similitude for shaking table tests on soil-structure-fluid model in $1 \mathrm{~g}$ gravitational field. Soils Found 29(1):105-118

17. Kramer SL (1996) Geotechnical earthquake engineering. Prentice Hall, Upper Saddle River

18. Seed HB, Idriss IM (1970) Soil moduli and damping factors for dynamic response analyses. College of Engineering University of California Berkeley, Berkeley 